\title{
Feature-based Product Modelling in Concurrent Engineering
}

\author{
T. De Martino and F. Giannini \\ Istituto per la Matematica Applicata, CNR \\ Via de Marini 6, I-16149 Genova, Italy \\ tel. +39106475666 \\ fax. +39106475660 \\ E-mail: demartino(giannini)@ima.ge.cnr.it
}

\begin{abstract}
Feature recognition, integrated with design-by-features, has the potential to support a multiple-view CAD environment, allowing the representation of different feature-based models, that are meaningful for different contexts. They represent a vital step in the direction of product modelling in Concurrent Engineering.

In this paper we present a flexible feature recognition system, in which topological, morphological and geometric information is processed at different levels of detail, providing the user with a mechanism that can be tuned to application needs.
\end{abstract}

\section{Keywords \\ Product Modelling, Feature-based Modelling, Feature Recognition, Concurrent Engineering}

\section{INTRODUCTION}

Nowadays, markets for consumer goods are characterised by increased variety coupled with a decrease in the product life-cycle. To face competition, it is important to optimise performance in delivery and quality.

The main goal of concurrent engineering (CE) is to reduce the costs incurred in product development by considering design, installation, organisation and control of production activities as a whole, and in such a way that all the decisions to be taken can be evaluated in relation with each other during the design phase. This new paradigm assumes an ideal environment, where engineers from different disciplines simultaneously work on product design. After an initial conceptual 
model has been defined, several engineers should be able to work at the same time on product definition, modifying or adding details to the design and also running application programs (e.g. stress analysis, cost analysis, process planning).

The implementation of $\mathrm{CE}$ requires a computer support not only provides a comprehensive framework for dealing with product models, but can also represent, transmit, manipulate and store all the technological information associated with the different phases of the product life cycle.

Traditional Computer Aided Design (CAD) systems, based purely on geometric and topological information, are unable to fulfil this aim, as they do not include any functional and behavioural information on the parts, nor do they provide facilities for representing different context-dependent views of the product, their interrelations and dependencies.

More recent CAD systems adopt feature-based modelling that enriches product data representations with semantic information and supports design with high level modelling entities. However, these systems are still far from satisfying industry's need to support an automatic flow from design to production in a CE environment, in part due to the context dependency of features. To improve CAD systems in this direction, current research proposes the integration of feature recognition techniques with the design-by-features approach in order to provide multiple context dependent views of the product simultaneously.

In this paper the need for feature-based product modelling with concurrent engineering is outlined. The benefits and usefulness of different techniques for feature-based modelling are discussed. Finally, a feature recognition system which has been integrated with a design-by-features system is described.

\section{FEATURES IN PRODUCT MODELLING}

Features have been introduced in the engineering field as elements providing a convenient language for describing product parts, one that associates functional meaning to geometry and thus allows each application to have its own view of the product (Shah and Mäntylä 1995). It is commonly accepted that a product model suitable for running application analysis and simulations should be based on features.

In the literature, two main approaches to the creation of feature-based representations have been considered: design-by-features and feature recognition. The former is a top-down approach: the object is designed directly using features and all the functional knowledge related to a specific context and strictly associated to the features used can be inserted in the model at the design stage, (Wilson and Pratt, 1988), (Shah, 1991), (Ovtcharova and Haßinger, 1991), (Schulz and Schützer, 1994). Since this approach provides a more natural basis of interaction with the user than mere geometric modellers, most commercial CAD systems presently on the market have adopted the design-by-features approach. As a matter of fact these systems are not really feature based, since the features they handle are simple, pre-defined parametric primitives corresponding to recurring 
characteristics of products (examples are shown in Figure 1), but no consistency check is performed after the modelling operations.

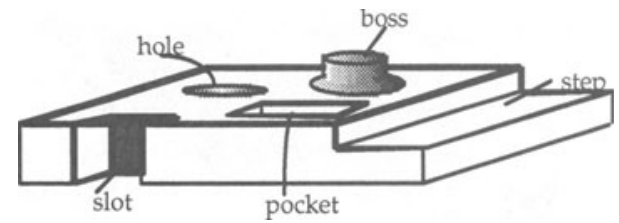

Figure 1: Examples of features

The disadvantage of this approach is that, since features are context dependent, when considering a different context it is necessary to redesign the object from scratch using a different and appropriate set of features (Dixon and Libardi, 1990).

The second approach is bottom-up: all the features are extracted from the geometry, thus making it possible to consider different applications by changing the recognition rules. This approach has mainly been mainly explored at the research level: (Vandenbrande and Requicha, 1993), (Joshi, 1990), (Falcidieno and Giannini, 1989), (Sakurai and Chin, 1994), (Regli et al. 1993). Only very recently some commercial systems propose modules for machining feature recognition from the geometric description of the part, PART ${ }^{\mathrm{TM}}$ from the original work of Van Houten (1991) and CAMWorks ${ }^{\mathrm{TM}}$. These modules perform semi-automatic feature recognition of the machining features; moreover they determine the operation sequence by consulting a previously defined database where each feature type is associated to a machining operation sequence. While these systems are most successful with quite simple geometric components, they nevertheless show the great potential of feature recognition for the CAD/CAM market.

The main problems of feature recognition appears in the recognition of interacting features, the reason being that when features interact, their canonical description is compromised, i.e. feature faces are split or merged into other object faces during model creation. Moreover, feature interaction gives rise to ambiguous configurations which can generate multiple object interpretations in terms of features. This problem requires the definition of strategies for determining the original entities in accordance with the requirements of a specific application context; volume creation may provide a solution here.

\section{FEATURE-BASED MODELLING FOR CE}

In $\mathrm{CE}$ different experts from different engineering disciplines participate simultaneously in the product design: they perform all kinds of analysis and make product enhancements; at the same time functional aspects of other disciplines must be preserved (de Kraker, 1998). From the design point of view this means that different feature-based representations of the product, containing the features relevant for the different experts, should be considered and kept consistent during the design evolution. 
An integrated feature-based modelling system, in which both feature design and recognition are possible, seems promising as a support to $C E$. In such a system the user can either design-by-features or use the solid moduller, depending on the complexity of the object; feature recognition techniques can be integrated in the core of the system in order to derive different feature-based models for specific application contexts and maintain consistency between them. In this way it is possible to create a link between the different application contexts and the design process, with the advantage of providing the designer with earlier feedback from the simulation processes and analysis performed in the specific contexts.

A feature-based modelling system which integrates design-by-features and feature recognition approaches has been developed at IMA-CNR in co-operation with Fraunhofer IGD, in Darmstadt, Germany, within the European HCM Project ERB CHBG CT930380 (De Martino et al. 1998).

This Integrated System is based on the following key points:

1) Feature recognition can serve multiple functions in integrated feature-based modelling systems. Three main applications have been studied: the traditional use of feature recognition from boundary representations for upgrading already existing solid models to feature-based ones; for the conversion of a design feature-based model into another one meaningful for a given application context; and for the validation of feature-based model consistency needed during the design process

2) To achieve integration between design and engineering processes, the sharing of a common product model representation is crucial. Such a model should be a multiple view model shared by the application modules, where all the contextdependent feature-based models derived by the feature recognizer are represented. The advantage of a single shared structure is that it favours communication between the application contexts, and in particular the negotiation and propagation of the modifications required during the design within the perspective of a collaborative design process.

The application scenario where design and engineering processes are integrated is shown in Figure 2. The designer creates the object using design features and defines the design model of the object. The design information is evaluated and stored in the shared model, called the Intermediate Model (IM), in such a way as to maintain the feature semantics explicitly associated to the shape description. From the data stored in the IM, the feature recognition process derives different featurebased models for different application contexts. In the specific contexts, experts can add additional context-dependent information to these models, run simulation programs and call for design modifications. In order to avoid conflicts among modification requests and to keep the different models consistent, the designer is the only user allowed to modify the object model. The experts' requests are sent via the IM to the designer, who executes the modifications after negotiation among the contexts and according to context-dependent priority rules. Finally, the accepted modifications are performed, directly represented in the IM and propagated to each specific context-dependent view. Feature model validation 
based on a feature recognition technique is applied to each view in order to ensure the models' consistency after each modification.

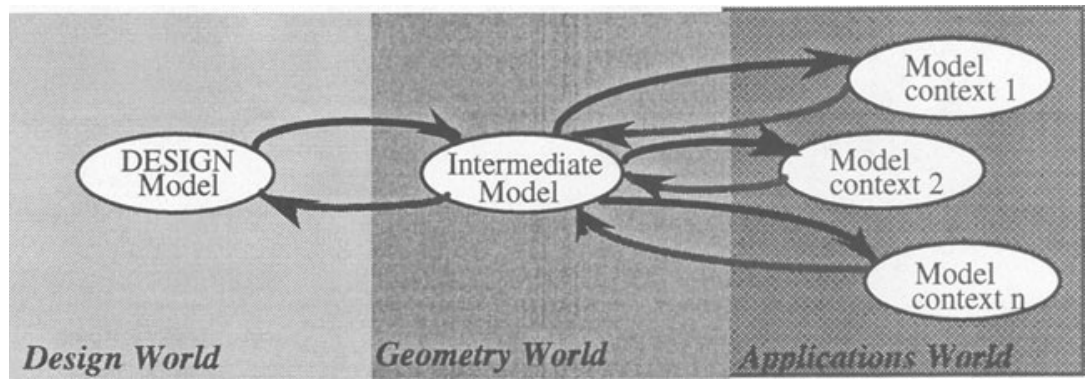

Figure 2: The application scenario of the integrated system

\section{THE FEATURE RECOGNISER}

The flexible feature recognition system is the result of many years of research at the Istituto per la Matematica Applicata, CNR, Genova. The main aim of this work is to provide the CAD user with a feature-recognition tool that can be tuned to meet the requirements of a specific application.

The methodology adopted in our approach is based on the differentiation between context-independent and context-dependent shape interpretation. The Boundary Representation of the product is analysed considering the geometric, morphological, and topological aspects separately, in order to identify neutral properties of the shape independent of any specific application context. These properties are then considered in the object context-dependent interpretation phase. Specifying the context, the user defines the set of features meaningful for the specific application that have to be recognised.

The recognition system consists of four main steps: Morphological Classification, Topological Classification, Evaluation of Geometric Relations, and context-dependent Feature Interpretation. The morphological classification analyses the B-rep of an object in order to identify face sets corresponding to generic concavities and convexities, interpreted as protrusion and depression shape features. Topological classification is then performed to identity topological characteristics of each shape feature. The evaluation of geometric relations between feature entities such as parallelism and perpendicularity between faces is then performed. Finally, the context dependent feature interpreter identifies specific feature instances by performing recognition at different levels of detail according to the user's requirements. This step may derive volume feature representations (if the specific context concerns volumetric features) and feature parameter values.

The data structure shared by these processes is the Intermediate Model, which stores all the information obtained step by step during the recognition process. A simplified system architecture is depicted in Figure 3. In the following sections the Recogniser components and the Intermediate Model are briefly described. 


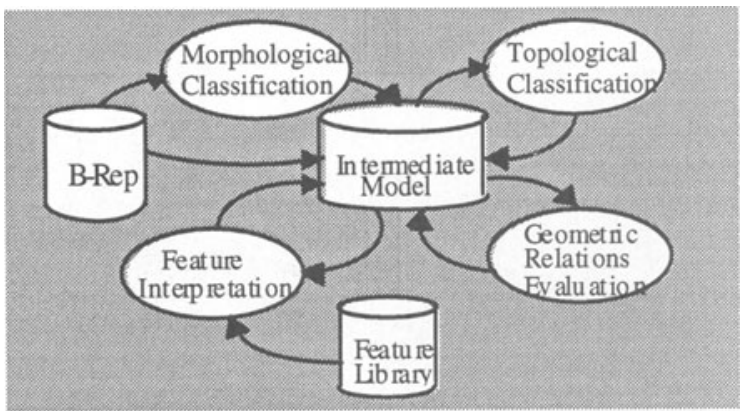

Figure 3: The Feature Recogniser architecture

\subsection{Morphological Classification}

The aim of this step is to identify generic protrusion and depression features by analysing the object boundary representation. Looking at the available feature taxonomies, it can be noted that simple features have a wholly concave /wholly convex shape, i.e. all the feature faces are adjacent to each other exclusively through concave (or convex) edges. Conversely, compound features have mixed concave and convex adjacencies. Therefore, the concepts of weak and strong concavity and convexity have been introduced and defined as follows:

- weak concavity (convexity): a set of connected faces, each adjacent to at least another face in the set through a concave (convex) edge;

- strong concavity (convexity): a set of connected faces whose internal edges (i.e. lying on two faces of the set) are concave (convex).

The process starts with the classification of edges and loops as either concave or convex. Then sets of faces belonging to weak characteristics are identified, thus decomposing the B-rep into face sets. Faces not belonging to any identified characteristic belong to the so-called main shape. Finally, strong characteristics are recursively and alternately sought within the identified face sets (see Figure 4).

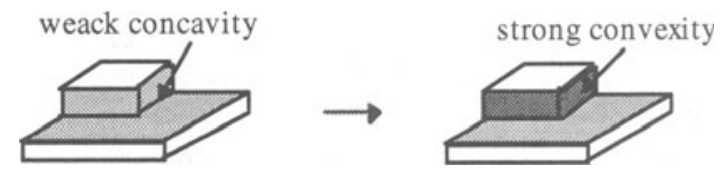

Figure 4: An example of weak and strong characterisation of a simple object. 


\subsection{Topological Classification}

In this module each shape feature identified in the previous step is analysed with the dual aim of studying invariant shape characteristics for the definition of shape feature equivalence relation, and providing feature interaction hints.

The definition of shape feature equivalence classes is based on the type of topological relationship between the feature and the rest of the object, and on the morphological properties of the feature's shape (De Martino and Falcidieno,1997). The aim is to define a feature classification independent of those shape variations which may affect a feature without changing its main properties. The elements that are evaluated are the so-called connection faces, faces of the object that are adjacent to feature faces, and the connectivity of the feature face set boundary. Figure 5 shows a set of equivalent shape features produced as a result of the classification.
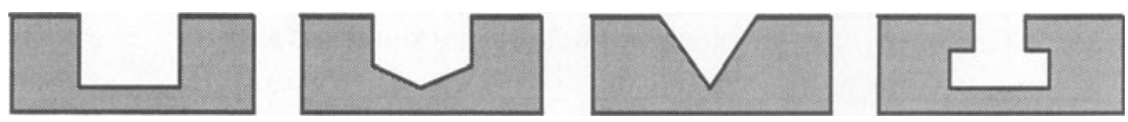

Figure 5: Examples of equivalent shape features.

The interaction hints are defined by analysing adjacency relationships among the feature face sets. They are expressed by an integer, called the interaction degree, which is related to the probability of interacting features, and, consequently, of multiple feature interpretations. Shape characteristics with degree 0 have only one possible interpretation. Conversely, shape characteristics with interaction hint degree $n, n>0$, always indicate the presence of feature interactions. Examples are shown in Figure 6.

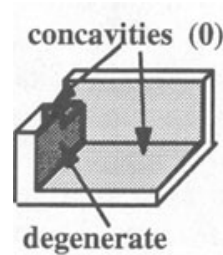

convexity (3)

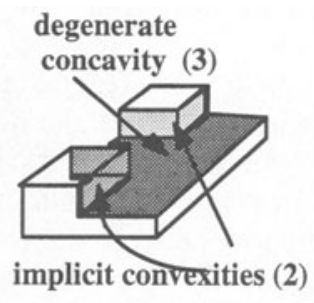

implicit convexities (2)

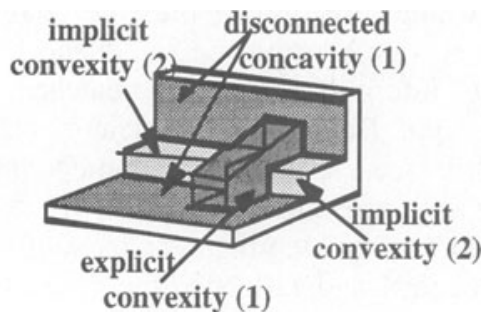

Figure 6: Examples of interaction hints.

\subsection{Geometric Relations Evaluation}

The aim of this step is to obtain a minimal set of geometric relations between feature entities, which characterises the feature shape and supports its parametric representation. The evaluation of geometric relations is performed for each feature and compreses two phases: first the set of all the relations involving the feature faces are derived, then the set is reduced by a simplification process. The 
geometric relations considered are parallelism, perpendicularity, incidence and coplanarity.

Three types of relations are derived: internal relations between pairs of feature faces, internal-external relations between one feature face and one connection face, and external-external relations between two connection faces of the feature. An example is shown in Figure 7.

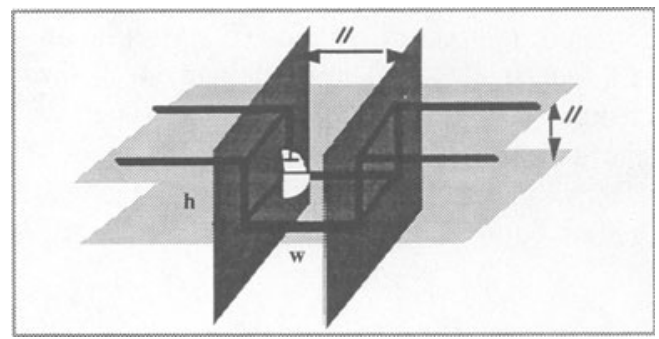

Figure 7: Geometric entity relations evaluation for a slot feature.

\subsection{Feature Interpretation}

The results of morphological classification, topological classification and evaluation of geometric relations express shape properties that characterise the features at different levels of detail. By reasoning about these results it is possible to define different conditions for defining shape similarity and for guiding the feature interpretation process.

The interpreter is based on a teaching-by-examples technique that uses the information stored in a feature library and performs a matching operation between the features in the library and the features in the object. Each application can have its own feature library, thus the recognition process is easily used for different contexts (De Martino and Falcidieno,1997).

The information that is matched regards the feature type (Main Shape, Protrusion, Depression), the feature classification result, the number of planar and quadric faces, as well as the number and type of the geometric entity relations. The matching operation between two features returns a number expressed as a percentage representing the degree of similarity between the two features. The user can control and influence the result by directly specifying an acceptance level. When the matching operation is successful, the interpreter performs the Parameter Matching: in this phase the set of parameters of the library feature are associated to the geometric entity relations of the feature of the object. The search for a specific feature instance can be concluded by checking the parameters value.

While the above interpretation works with surface feature representations, i.e. face sets, some applications deal with volumetric features. For this reason a process, performed only for some applications, for deriving feature volumes has been provided offering the possibility of considering only negative features, as in the case of machining, or also positive ones, which are useful in design. This results in the grouping/redescribing of several shape features depending on the associated interaction hints. The feature volume is obtained by intersecting two 
infinite volumes, which we call internal and external. The internal volume is obtained from the faces corresponding to the feature, while the external volume is derived from a subset of the neighbouring faces. The way of grouping shape characteristics to create the internal shape strictly depends on the selected environment (i.e. only depressions and/or protrusions), while the choice of the neighbouring faces depends on whether volumes corresponding to the intersection of the interacting features are also required. In addition, the module offers the possibility of controlling the determination of the volumes for complex interacting features. This process is based on some heuristic rules that take into account the interaction hints and the geometric relations among faces, like co-planarity and parallelism. In this way, it is possible to decompose the feature volumes and to allow multiple interpretations (Giannini, 1997). Figure 8 shows some examples of the possible derived volumes, the semplicity of the object is for clarity of the drawing and is due to lack of space.

\subsection{The Intermediate Model}

The Intermediate Model has been defined mainly to support multiple feature-based models, which is a crucial problem in Concurrent Engineering. It is necessary to consider an integrated product model where shape features for different contexts are represented in such a way as to avoid data redundancy, and to support consistency checks (De Martino, 1997). In particular, the Intermediate Model is the reference data model of the recogniser modules where all the obtained information is stored. The information contained in it regards the shape features of the product and their semantics from different points of view.

The shape features are explicitly represented in the model by their set of boundary entities and by the topological adjacency relations between feature pairs. This information is fundamental for performing geometric and topological reasoning on the shape, which is the basis of the feature recognition process.

The semantic representation of a feature is given by a set of relations between shape entities expressed in algebraic form. These relations are associated to and express the feature parameters and the set of geometric constraints necessary to control the behaviour of the feature during the design process. Shape representation and semantic representation are then unified by considering the correspondence and maintaining a link between the shape and the algebraic entities. 


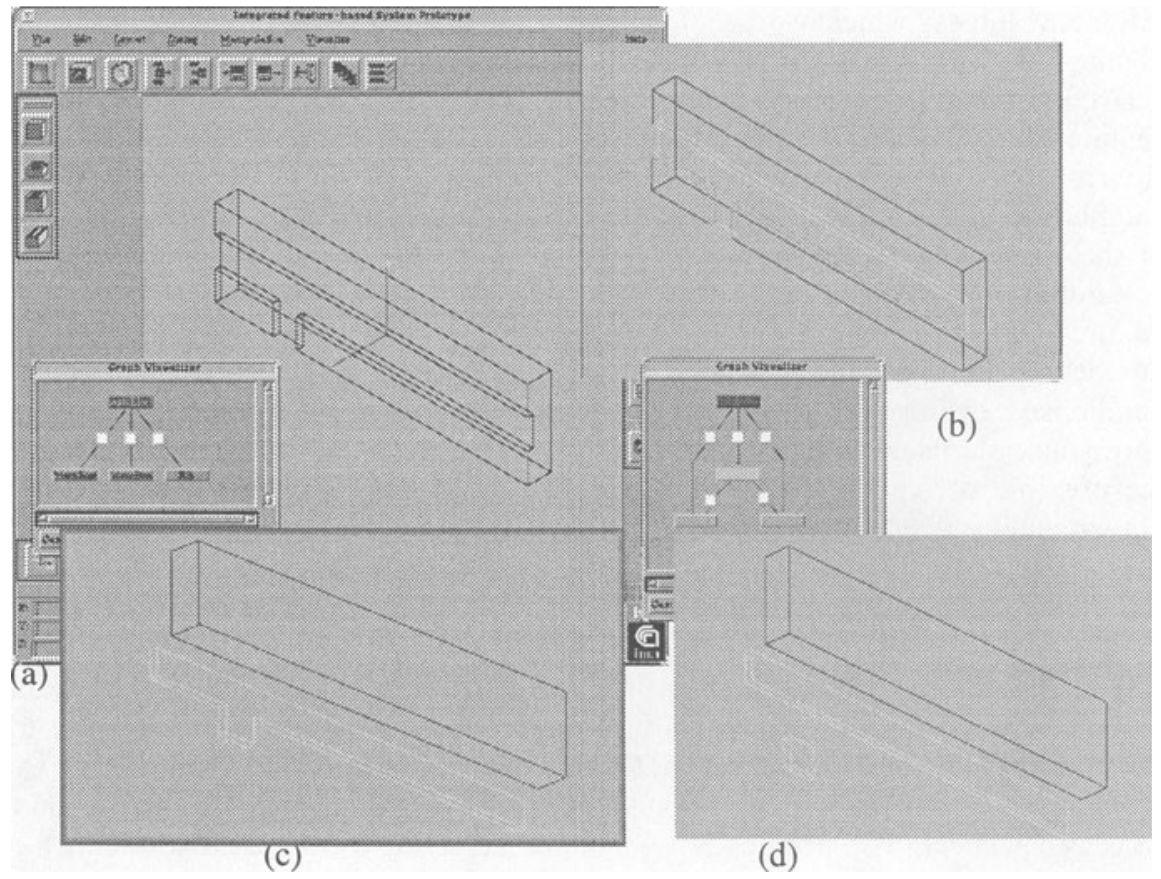

Figure 8: A simple object and its design graph (one rib and two prismatic bosses) (a); the result of the shape recognition: two convexities in green and one concavity in yellow (b); and two possible feature volume creations (c) and (d)

\section{CONCLUSIONS}

In this paper, the importance of feature-based modelling in CE has been outlined together with the necessity of integrating design-by-features and feature recognition for supporting the multiple product views needed for driving downstream applications and analysis. A flexible recognition system suitable for deriving different context dependent features has been presented. This system has been implemented on the ACIS ${ }^{\mathrm{TM}}$ solid modeller toolkit, and has been integrated with a design-by-feature system. To make the propagation of modifications among contexts easier, a multi-view model has been used; this is shared by the considered contexts and provides information about the object entities of interest to them. At present, the only context with the authority to modify the object is design; the others can only ask for modifications. The system is based on differentiation between context independent shape analysis and context dependent feature interpretation, which also provides the possibility of deriving both surface and volumetric feature descriptions. This offers several advantages: the creation of the most suitable representation for context needs; easier re-use of existing models created by traditional solid modellers; the manipulation of features by the other 
contexts, i.e. object modification through feature parameter changes; and the identification of simple features in interacting configuration. In particular, some of the possible descriptions of simple features in interacting configurations can be provided.

Future work will focus on the optimisation and extension of the volume determination algorithm and on support of the negotiation activity for product modifications.

\section{REFERENCES}

ACIS $^{\mathrm{TM}}$ (1993) Geometric Moduller, Spatial Technology, Boulder Colorado CAMWorks ${ }^{\mathrm{TM}}$ (1997) from Teksoft ${ }^{\mathrm{TM}}$ CAD/CAM systems

de Kraker, K.J. (1998) Feature conversion for concurrent engineering, $P h D$ thesis, Technische Universiteit Deft, ASCI N 21

De Martino, T. (1997) A Multiple-View CAD Representation for Product Modeling, Proceedings of Shape Modeling International '97, International Conference on Shape Modeling and Applications, Aizu-Wakamatsu, Japan

De Martino, T. and Falcidieno, B. (1997) A Tuning mechanism for Feature Recognition, Proc. of the ASME DFM Symposium '97, Sacramento, USA

De Martino, T., Falcidieno, B. Haßinger, S. (1998) Design and Engineering Process Integration through a Multiple View Intermediate Moduller in a Distributed Object-Oriented System Environment, to appear on Computer Aided Design, special issue on Network-Centric CAD

Dixon, JR., Libardi, EC., (1990) Unresolved Research Issues in Development of Design with Features Systems, in: Geometric Modeling for Product Engineering, North Holland, IFIP1990, pp. 183-196

Falcidieno, B., Giannini, F. (1989) Automatic recognition and representation of shape-based features in geometric modeling systems, Computer Vision, Graphics and Image Processing, 20, pp. 93-123

Giannini, F. (1997) Context Dependent Extraction of Volumetric Features, Proceedings of Shape Modeling International '97, International Conference on Shape Modeling and Applications, Aizu-Wakamatsu, Japan

Joshi, S. (1990) Feature recognition and geometric reasoning for some process planning activities, Geometric Modeling for Product Engineering, M.J.Wozny, J. Turner, K. Preiss Editors (IFIP '88), pp. 363-383

Ovtcharova, J., Haßinger, S. (1991) Feature-Based Reasoning in Product Modeling, Proc. of IFIP TC5 Conference on Computer Applications in Production and Engineering, Integration Aspects, CAPE'91, Bordeaux, France, pp.379-387

PARTTM (1998) Tecnomatix Technoogies Ltd..

Regli, W.C., Gupta, S.K., Nau, D. (1993) Building a general approach to feature recognition of material removal shape element volumes, Sec. Symposium on Solid Modeling Fundations and CAD/CAM applications, ACM press

Sakurai, H. , Chin, C.W. (1994) Definition and recognition of volume features for process planning, J.J. Shah, M. Mäntylä and Dana Nau eds., Advances in Feature BasedManufacturing, pp. 65-80, Elsevier/North Holland 
Shah J.J., Mäntylä M. (1995) Parametric and Feature-based CAD/CAM: concepts, techniques and applications, Wiley Interscience

Shah, J.J., (1991) Assessment of features technology, Computer-Aided Design Vol. 23 , No. 5, pp. 331-343

Schulz, H., Schützer, K. (1994) FINDES-Integrating design and manufacturing, IFIP International Conference: Feature Modeling and Recognition in Advanced CAD/CAM Systems, Valenciennes

Vandenbrande, J.H., Requicha, A.A., (1993) Spatial Reasoning for the automatic recognition of machinable features in solid models, IEEE Transaction on Pattern Analysis and Machine Intelligence, 15 (12), pp. 1269-1285

Van Houten, F. J. A. M. (1991) PART: A computer Aided Process Planning System, PhD Thesis, University of Twente

Wilson, P.R., Pratt, M.J.(1988), A taxonomy of Features for Solid Modeling, Geometric Modeling for CAD Applications, M.J.Wozny, H.W.McLaughlin, J.L.Encarnaçao Eds., Proc. IFIP WG5.2 Conf., Albany, NY, North Holland

\section{BIOGRAPHY}

Teresa De Martino is currently a Senior Researcher at the Istituto per la Matematica Applicata of the Italian National Council of Research, Genova, Italy, where she began to work in 1990. From 1993 to 1996 she was guest researcher at the Department for Industrial Applications at the Fraunhofer Institut Graphische Datenverarbeitung in Darmstadt, Germany. She holds a degree in Mathemathics from the University of Genova (1990) and a Ph.D. in Computer Science (1998) from the Technische Universität of Darmstadt, Germany.

Her research interests include Geometric Modelling, Feature-based Modelling, and Product Modelling with Concurrent Engineering.

Franca Giannini is Researcher at the Istituto per la Matematica Applicata of the Italian National Council of Research, Genova, Italy, where she has been working since 1989. She got the degree in Mathemathics from the University of Genova in 1986. From 1986 to 1989 she worked for Italcad. She has been involved in several national and international projects on product modeling and production automation. She is also a co-author of two patented systems. Her research interests include Geometric Modelling, Feature-based Modelling, and Product Modelling with Concurrent Engineering. 